\title{
«Peut-on en avoir un peu plus?»
}

Non, désolé, mais je reviendrai là-dessus un peu plus tard ...

Je me souviens parfaitement des grands débats menés sur les suppléments et forfaits pour urgence qui ont finalement été inscrits dans le TARMED. Deux constatations à ce sujet: d'une part, le concept d'urgence a été défini de manière très stricte dans le tarif. Cette définition ne laisse pratiquement aucune marge de manœuvre à qui l'utilise de manière conséquente et correcte. D'autre part, les indemnisations en francs promises pour les forfaits d'urgence n'ont jamais été réalisées ou ont été tout simplement «escamotées».

Pour les consultations données le dimanche à des patients qui ne sont pas de «vraies» urgences, nous ne pouvons ainsi taxer la prestation fournie que comme un jour de semaine. Ce fait a conduit la SSMG et la FMH à prendre en mains le problème dès 2004. Le résultat est là, qui ne semble pas satisfaire tout le monde.

Comme cela avait été prévu, la délégation aux négociations (avec des représentants de la FMH et de la SSMG) a négocié avec les assureurs une «indemnité forfaitaire de dérangement pour consultation pressante en dehors des heures régulières de consultation, ainsi que le soir et le week-end». Cette indemnité a été acceptée par TARMED Suisse (qui rassemble la FMH, santésuisse, la CTM, H+, et la CDS en tant qu'observatrice). Depuis lors, le Conseil fédéral l'a aussi approuvée en demandant aux protagonistes d'observer cette nouvelle position jusqu'à fin 2007 quant à ses objectifs et à ses coûts, et de proposer des modifications selon les cas.

Un forfait pour urgence pendant la consultation n'aurait aucune chance de franchir le barrage des instances responsables. De plus, une telle position entraînerait un fort potentiel d'abus et pourrait conduire, suite à une «ex- tension des prestations» pendant la phase de la convention prestations-prix, à une baisse de la valeur du point tarifaire dans les cantons.

Avant d'adopter des modifications de la structure tarifaire du TARMED, le Conseil fédéral demande que celles-ci soient neutres en termes de coûts. Ce fut le cas également des forfaits pour consultation pressante. Il ne les aurait jamais approuvés s'ils avaient dû générer des dépenses supplémentaires. La délégation aux négociations a donc dû en tenir compte, ce qui a conduit en conséquence à abaisser les forfaits d'urgence $\mathrm{A}$ à $\mathrm{C}$. Il n'était pas question, dès lors, d'en «avoir un peu plus»!

La délégation FMH/SSMG avait ainsi deux possibilités: soit accepter d'introduire les forfaits pour consultation pressante en respectant la neutralité des coûts, ou alors laisser les choses comme elles étaient. La décision qui fut prise, et le fut en commun, est connue de tous.

Il est important que nous continuions à appliquer correctement la notion d'urgence et que nous ne développions pas, par dépit, nos propres interprétations. Ceci ne conduirait sûrement pas à une amélioration. Ce qui est essentiel est de suivre la situation en ce qui concerne ces indemnités pour urgence et pour consultation pressante et, fin 2007, d'analyser la situation et de présenter alors des demandes en vue d'obtenir des corrections.

Par la création du Bureau des tarifs au sein de son domaine Tarifs et conventions, la FMH a créé un instrument qui donnera une base encore plus large aux futures décisions en matière tarifaire. Le Bureau des tarifs réunit en son sein des sociétés faîtières (FMCH, FMPP, SFSM), la Conférence des sociétés cantonales de médecine (CCM) et le domaine Tarifs de la FMH.

Dr Ernst Gähler, membre du Comité central de la FMH

\section{Questionnaire dans le cadre d'une procédure concernant le Viagra, le Levitra et le Cialis: information à I'intention des médecins contactés par la Comco}

Les médecins qui ont reçu un courrier de la Commission de la concurrence trouveront des informations du VEDAG à ce sujet sur le site internet de la FMH (www.fmh.ch; sélectionner la langue, l'information est ensuite directement visible). 